\title{
In Addition to the High-Density Lipoprotein Fraction, Apolipoprotein C-III is Detected in Chylomicrons and the Very Low-Density Lipoprotein Fraction from Serum of Normolipidemic Cows
}

\author{
Norio KATOH ${ }^{1)}$ \\ ${ }^{1)}$ Hokkaido Research Station, National Institute of Animal Health, 4 Hitsujigaoka, Sapporo 062-0045, Japan
}

(Received 7 August 2000/Accepted 6 September 2000)

ABSTRACT. Apolipoprotein (apo) C-III is a low-molecular-mass protein that is involved in the regulation of the triglyceride metabolism. Except for the hyperlipidemic calf, cattle apoC-III is mainly detected in the high-density lipoprotein (HDL) fraction, and the distribution in chylomicrons (CM) and the very low-density lipoprotein (VLDL) fraction has not yet been clarified. The purpose of the present study was to detect apoC-III in concentrated CM and VLDL fractions to examine whether apoC-III is distributed in the two fractions even in normolipidemic cattle. ApoC-III could be detected by immunoblot analysis in both concentrated cow CM and VLDL fractions, but not in the corresponding calf fractions. These results suggest that apoC-III is distributed in the CM and VLDL fractions, at least in cows, although the concentrations in these fractions are considerably lower than in the HDL fraction.

KEY WORDS: apolipoprotein C-III, bovine, chylomicron, high-density lipoprotein, very low-density lipoprotein.

J. Vet. Med. Sci. 63(1): 95-97, 2001

Apolipoprotein (apo) C-III, the low-molecular-mass protein mainly synthesized by the liver, is distributed in human plasma in chylomicrons (CM), very low-density lipoprotein (VLDL) and high-density lipoprotein (HDL) fractions. The apoC-III is associated with HDL in the fasting state, but it is transferred to the CM and VLDL fractions after the plasma triglycerides (TG) concentration is increased by absorption of dietary fat [1]. The total plasma concentration of apoC-III increases in patients with hypertriglyceridemia [3]. In mice, overexpression of apoC-III gene results in hypertriglyceridemia [2], whereas disruption of the apoC-III gene induces hypotriglyceridemia [4], thereby suggesting that apoC-III is involved in the regulation of metabolism of TG. In contrast to its human counterpart, cow apoC-III is mainly detected in the HDL fraction, not in the CM and VLDL fractions [10]. The re-distribution from HDL to the CM and VLDL fractions by feeding is not observed, probably because of the indistinct alimentary lipemia in cattle. An exceptional case of the distribution of apoC-III in the CM (higher concentration than in the HDL fraction) was found in a calf with hypertriglyceridemia [12]. The purpose of the present study was to examine whether apoC-III was distributed in the CM and VLDL fractions from normolipidemic cattle. For this purpose, immunoblot analysis, the most sensitive method for detection of apoC-III [11], was applied to CM and VLDL fractions which had been extensively concentrated. The distribution of apoB-100 was also examined as a marker for VLDL.

Sera from 3 Holstein cows during midlactation (3 to 5 years old) and 3 male Holstein calves ( 2 to 5 days after birth) were used. Blood was collected in the morning (before feeding). Onto 32 to $44 \mathrm{~m} l$ of serum, a half volume of a density solution $(d=1.006 \mathrm{~g} / \mathrm{m} l,[8])$ was layered, and centrifuged at $26,000 \times \mathrm{g}$ for $30 \mathrm{~min}$. The top layer (1/6 of total volume) was aspirated, and the next $1 / 6$ layer was discarded. The col- lected fraction was washed twice by mixing it with 5 volumes of the density solution and centrifuging this mixture at $26,000 \times \mathrm{g}$ for $30 \mathrm{~min}$, and used as the $\mathrm{CM}$ fraction. Onto the remaining solution after the first centrifugation, a half volume of the density solution was layered, centrifuged at $114,000 \times \mathrm{g}$ for $16 \mathrm{hr}$, and the top 1/6 layer was collected as the VLDL fraction. The CM and VLDL fractions were dialyzed against phosphate-buffered saline (PBS), and thereafter concentrated by an Amicon YM-10 (Millipore Corp, Bedford, MA, U.S.A.). Low-density lipoprotein (LDL) and HDL fractions were prepared from $4 \mathrm{~m} l$ each of cow and calf sera [8]. ApoC-III was purified from cow serum [10]. AntiapoC-III [11] and anti-apoB-100 [13] were raised in rabbits. Immunoblot analysis was performed as described previously [11]. Detection limits of the analysis were approximately 10 $n \mathrm{~g}$ for apoC-III and $100 \mathrm{ng}$ for apoB-100 under the conditions used. Sodium dodecyl sulfate-polyacrylamide gel electrophoresis (SDS-PAGE) was done using a Tricine-buffer system for apoC-III [10] and a glycine-buffer system for apoB-100 [8]. In the electrophoresis for apoB-100, a 5-20\% gradient gel was used. The serum TG concentration was measured enzymatically.

SDS-PAGE and subsequent Coomassie Brilliant Blue staining of cow lipoprotein fractions revealed that apoC-III, apoA-I, apoA-IV and a $35 \mathrm{kDa}$ protein were mainly distributed in the HDL fraction, whereas apoB-100 was in the LDL fraction (Fig. 1). In the CM and VLDL fractions, except for serum albumin, bands of apolipoproteins including apoC-III and apoB-100 were not obviously observed. SDS-PAGE of calf lipoprotein fractions gave similar results (figure not shown).

ApoC-III was detected in HDL fractions from cow and calf sera by immunoblot analysis (Fig. 2). The apoC-III band in cow HDL was denser than that in calf HDL, reflecting the higher apoC-III concentration in cows than in calves $[9,10]$. 


\section{.}

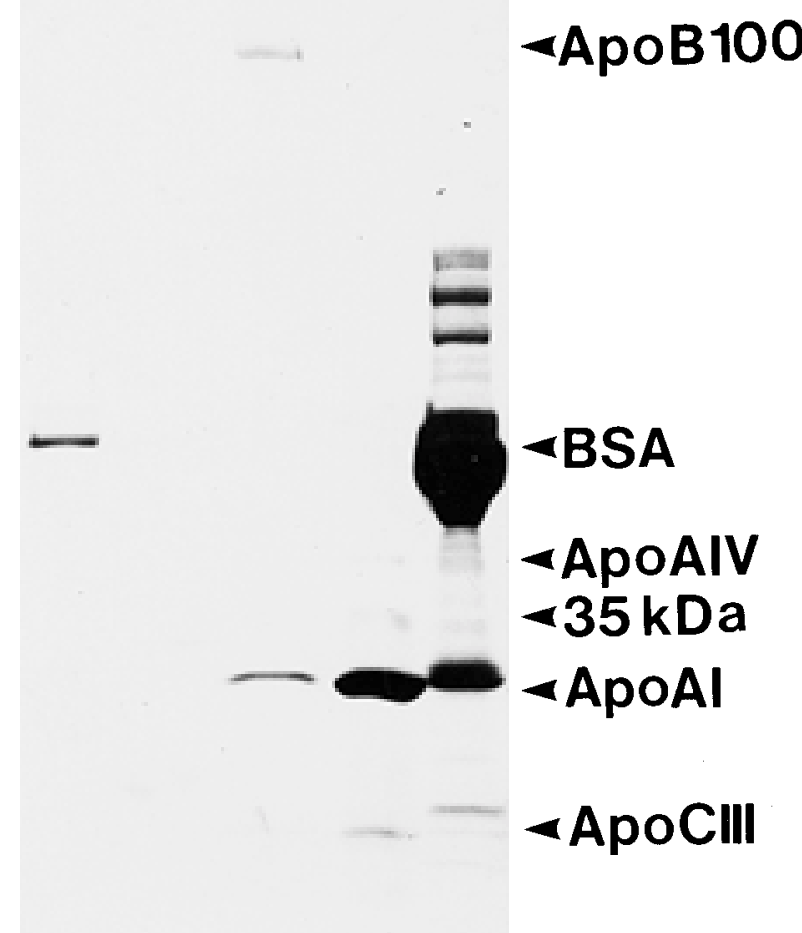

Fig. 1. SDS-PAGE of cow lipoprotein fractions. Undiluted lipoprotein fractions and 20-fold-diluted serum with PBS were mixed with a half volume of the SDS-sample buffer and boiled for $3 \mathrm{~min}$, and a $10 \mu l$ aliquot was applied to each well of a 5-20\% gradient gel. After electrophoresis, the gel was stained with Coomassie Brilliant Blue. BSA, bovine serum albumin.

Neither cow nor calf apoC-III was detectable in the original (unconcentrated) CM and VLDL fractions even by use of immunoblotting. The cow apoC-III band became apparent when CM and VLDL fractions were concentrated, but the calf band did not.

From the densitometric data, together with concentrations of samples and electrophoretic conditions (dilution of HDL and volumes applied to wells), apoC-III concentrations in original CM and VLDL fractions (means of 3 estimations) were calculated to be $0.0924 \mu \mathrm{g} / \mathrm{m} l$ and $0.158 \mu \mathrm{g} / \mathrm{m} l$, respectively. These values were approximately $1 / 1,100$ and $1 / 650$, respectively, of the HDL apoC-III concentration $(102 \mu \mathrm{g}$ / $\mathrm{ml}$ ). Bands for apoB-100 were visible in the original VLDL fractions (lanes 3 and 8), but not in the CM fractions (lanes 1 and 6). Both cow and calf apoB-100 bands were detected in the concentrated $\mathrm{CM}$ fractions, and the bands in the concentrated VLDL fractions became more distinct than in the original fractions. Serum TG concentrations were in the range of $10-30 \mathrm{mg} / 100 \mathrm{ml}$. TG concentrations were higher in calves than in cows, but were not significantly different.

The detection of apoC-III in both CM and VLDL fractions was not attributable to cross-contamination of the two fractions because apoB-100 was distinctly detected in cow and

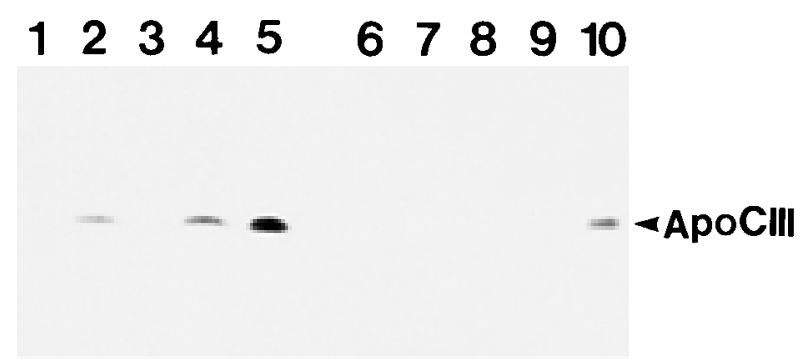

123456678910

Fig. 2. Detection by immunoblot analysis of cow and calf apoC-III and apoB-100 in concentrated CM and VLDL fractions. Lanes and protein concentrations $(\mathrm{mg} / \mathrm{m} l)$ are: 1 , cow original CM (0.341): 2, cow concentrated CM (3.18); 3, cow original VLDL (0.142); 4, cow concentrated VLDL (1.04); 6, calf original CM (0.532); 7, calf concentrated CM (1.59); 8, calf original VLDL (0.259); and 9, calf concentrated VLDL (0.729). Ten-fold-diluted cow HDL (upper lane 5), calf HDL (upper lane 10), cow LDL (bottom lane 5) and calf LDL (bottom lane 10) were included as positive controls. Densities are: apoC-III, 1 (ND, not detected); 2 (42); 3 (ND); 4 (57); 5 (100); 6 (ND); 7 (ND); 8 (ND); 9 (ND); and 10 (69): apoB-100, 1(ND); 2 (24); 3 (42); 4 (348); 5 (100); 6 (ND); 7 (13); 8 (125); 9 (401); and 10 (26). Volumes applied were $10 \mu l$ for cow fractions, $20 \mu l$ for calf fractions, $2 \mu l$ for HDL, and $10 \mu l$ for LDL. Analysis of CM and VLDL fractions from 2 other cows and 2 other calves gave similar results.

calf VLDL, but faintly in the CM fractions. It is also unlikely that the VLDL apoC-III was due to contamination by LDL, because of the extremely low LDL apoC-III concentration, as indicated in Fig. 1 and in previous studies $[9,10,12]$. Other than the liver, apoC-III is synthesized by the small intestine [5]. The origin of CM apoC-III seemed to be the small intestine. The transfer from the HDL fraction is less probable, because blood was collected before feeding. Lipid contents in the cow diet are low (less than 5\%), and the major energy source for cows is volatile fatty acids produced in the rumen rather than long chain saturated fatty acids absorbed by the intestine [6]. This unique lipid metabolism appears to be the reason for the extremely low apoC-III concentration in the $\mathrm{CM}$ fraction. Cattle have another characteristic with respect to hepatic VLDL secretion, which is considerably low compared with nonruminants [7]. The low apoC-III concentration in the VLDL fraction may reflect the limited VLDL secretion in cattle.

We have previously shown that apoC-III concentrations of calves one to three months old (20-40 $\mu \mathrm{g} / \mathrm{ml}$ [9]) are lower than those of lactating cows (60-120 $\mu \mathrm{g} / \mathrm{m} l$ [11]). Indeed, calf HDL apoC-III showed lower density than cow HDL 
(Fig. 2). ApoC-III concentrations in calf fractions seemed to be too low to detect by immunoblotting, even after concentration by the Amicon YM-10 membrane. The cut-off value of this membrane is approximately $10 \mathrm{kDa}$, while molecular masses of apoC-III are 8.2 and $7.3 \mathrm{kDa}$ (the latter is the major species) [10]. ApoC-III in HDL is not filtrated by the YM-10 membrane unless HDL is delipidated. The calf apoB-100 concentration is also lower than that in cows (Oikawa, unpublished results), but its content, particularly in the VLDL fraction, was sufficient for detection by immunoblot analysis. The apoB-100 band migrated more slowly than a $250 \mathrm{kDa}$ marker protein, indicating that this band was not apoB-48. The apoB-48 band was not clearly seen. The reason for this is not known.

In conclusion, apoC-III was shown to be distributed in the CM and VLDL fractions at least in cows. The distribution in these fractions suggests that cow apoC-III is synthesized, although to a lesser extent, by the small intestine, and also that liver apoC-III is secreted with VLDL as well as with HDL. The lower apoC-III concentrations in the CM and VLDL fractions appear to reflect the unique properties of the lipid metabolism in cattle.

ACKNOWLEDGEMENTS. The author is grateful to Ms. R. Nunome for her helpful advice on densitometry and to $\mathrm{Mr}$.
M. K. Barrymore for his critical reading of the manuscript.

\section{REFERENCES}

1. Havel, R. J., Kane, J. P. and Kashyap, M. L. 1973. J. Clin. Invest. 52: 32-38.

2. Ito, Y., Azrolan, N., O'Connell, A., Walsh, A. and Breslow, J. L. 1990. Science 249: 790-793.

3. Kashyap, M. L., Srivastava, L. S., Hynd, B. A., Gartside, P. S. and Perisutti, G. 1981. J. Lipid Res. 22: 800-810.

4. Maeda, N., Li, H., Lee, D., Oliver, P., Quarfordt, S. H. and Osada, J. 1994. J. Biol. Chem. 269: 23610-23616.

5. Mahley, R. W., Innerarity, T. L., Rall, S. C. and Weisgraber, K. H. 1984. J. Lipid Res. 25: 1277-1294.

6. Palmquist, D. L. 1976. J. Dairy Sci. 59: 355-363.

7. Pullen, D. L., Liesman, J. S. and Emery, R. S. 1990. J. Anim. Sci. 68: 1395-1399.

8. Uchida, E., Katoh, N. and Takahashi, K. 1992. Am. J. Vet. Res. 53: 2035-2042.

9. Yamamoto, M. and Katoh, N. 2000. J. Vet. Med. Sci. 62: 49-52.

10. Yamamoto, M., Katoh, N., Adachi, Y. and Oikawa, S. 1998. Am. J. Vet. Res. 59: 667-672.

11. Yamamoto, M., Katoh, N. and Oikawa, S. 1998. Am. J. Vet. Res. 59: 1358-1363.

12. Yamamoto, M., Oohashi, T., Katoh, N. and Oikawa, S. 2000. J. Vet. Med. Sci. 62: 1033-1039.

13. Yamamoto, O., Oikawa, S. and Katoh, N. 1995. Am. J. Vet. Res. 56: 1413-1417. 